\title{
Transmission Electron Microscopy of Coherence and Nanothermodynamic Effects in Graphene Biased In Situ
}

\author{
William A. Hubbard, Matthew H. Mecklenburg, E. R. White, Alexander Kerelsky, and B. C. Regan \\ Department of Physics \& Astronomy and California NanoSystems Institute, University of California, \\ Los Angeles, California 90095 USA
}

Graphene, a monolayer of carbon atoms forming a honeycomb lattice, has become the premier material in the exploration of two-dimensional systems and nano-electronics. With applications encompassing ultra-sensitive sensors, super-capacitors, and nano-circuitry, there is considerable interest in understanding the electrical and thermal behavior of graphene at the nanoscale. Here we report on the fabrication and characterization of graphene-based electronic devices for in situ transmission electron microscopy (TEM) observation, as well as the development of new nano-characterization techniques.

We are currently developing a method for mapping temperature with nanoscale resolution based on electron diffraction. High-order peaks in a crystal's diffraction pattern are attenuated by thermal motion; this attenuation is parameterized by a Debye-Waller factor. An analytical calculation of electron diffraction from graphene provides an expression for this factor, enabling us to extract the Debye-Waller factor from its selected area electron diffraction (SAED) pattern [1]. From the Debye-Waller factor we extract the temperature.

For our experiments we transfer single-layer graphene, produced by micro-mechanical exfoliation on $\mathrm{Si} / \mathrm{SiO}_{2}$ chips, onto chips designed for in situ TEM biasing of devices. The biasing chips consist of a $\mathrm{Si} / \mathrm{SiO}_{2} / \mathrm{SiN}_{\mathrm{x}}$ wafer with pits $\mathrm{KOH}$-etched into the back side leaving thin, electron transparent $\mathrm{SiN}_{\mathrm{x}}$ windows on the top side, around which we pattern $\mathrm{Cr} / \mathrm{Au}$ electrodes. To transfer graphene sheets from $\mathrm{Si} / \mathrm{SiO}_{2}$ to biasing chips we apply a PMMA backing layer and release the graphene by etching the oxide layer with $\mathrm{NaOH}$. We then position the graphene sheet over the window of the biasing chips using micromanipulators, dry the chip, and finally remove the PMMA. The result is an electrically contacted single-crystal graphene monolayer sitting on an electron-transparent membrane.

The completed chip is loaded into a Hummingbird TEM stage customized for device biasing. All of our measurements are performed in a FEI Titan 80-300 FEG TEM, which is operated at $80 \mathrm{kV}$ to minimize knock-on damage to the carbon lattice. After initial characterization of the graphene sheet (highresolution imaging, diffraction, dark-field imaging), the sample bias is slowly increased while video of bright-field TEM is recorded. The sample is monitored visually and electronically as voltage across the sample is increased to a safe maximum bias. As we approach this bias value we see plateauing of current for a given voltage as annealing ceases and sublimation of the residual oxide on membrane at the "hot spot." A bright-field TEM image of a device at this stage is shown in Figure 1, along with a close-up of the hot spot (inset). Once a safe maximum bias is determined we collect bright-field and dark-field images as well as SAED patterns at various bias voltages in order to probe the nanothermodynamics of the system and asses the effects of the high-temperature Joule-heating.

The diffraction patterns to the right in Figure 1 were acquired from a region within the hot spot of the device shown to the left. The attenuation in the higher-order peaks can be seen with the naked eye; the peak encircled in red, for example, disappears almost completely when the graphene is Joule-heated at 
$3 \mathrm{~V}$. Our measurement of the Debye-Waller factor using this data shows that the mean-squared thermal displacement of atoms at the hot spot while biasing is 2.5 times greater than the zero bias (room temperature) value $\left(44 \mathrm{pm}^{2}\right)$. At constant bias, diffraction data collected from regions incrementally further from the center of the hot spot show a decrease in the thermal motion of the region probed. The resulting thermal map can be compared to single-color pyrometry of graphene-based devices [2].

This platform for in situ TEM biasing of graphene samples and the diffraction analysis enable unique modes of nanoscale inspection. We are able to thermally map graphene with unprecedented resolution, as well as corroborate this data with established pyrometry techniques to explore how nanometer-sized defects and contaminants affect graphene as a blackbody. The Debye-Waller approach to thermal mapping can also help to clarify the dynamics of low-dimensional phase transitions.

\section{References:}

[1] B. Shevitski, et al, Physical Review B 87 (2013), 045417

[2] S. Singer, et al, Physical Review B 84 (2011), 195468

[3] This work was supported by NSF grants DMR-0748880 and DMR-1206849, and the Electron Imaging Center for NanoMachines at UCLA

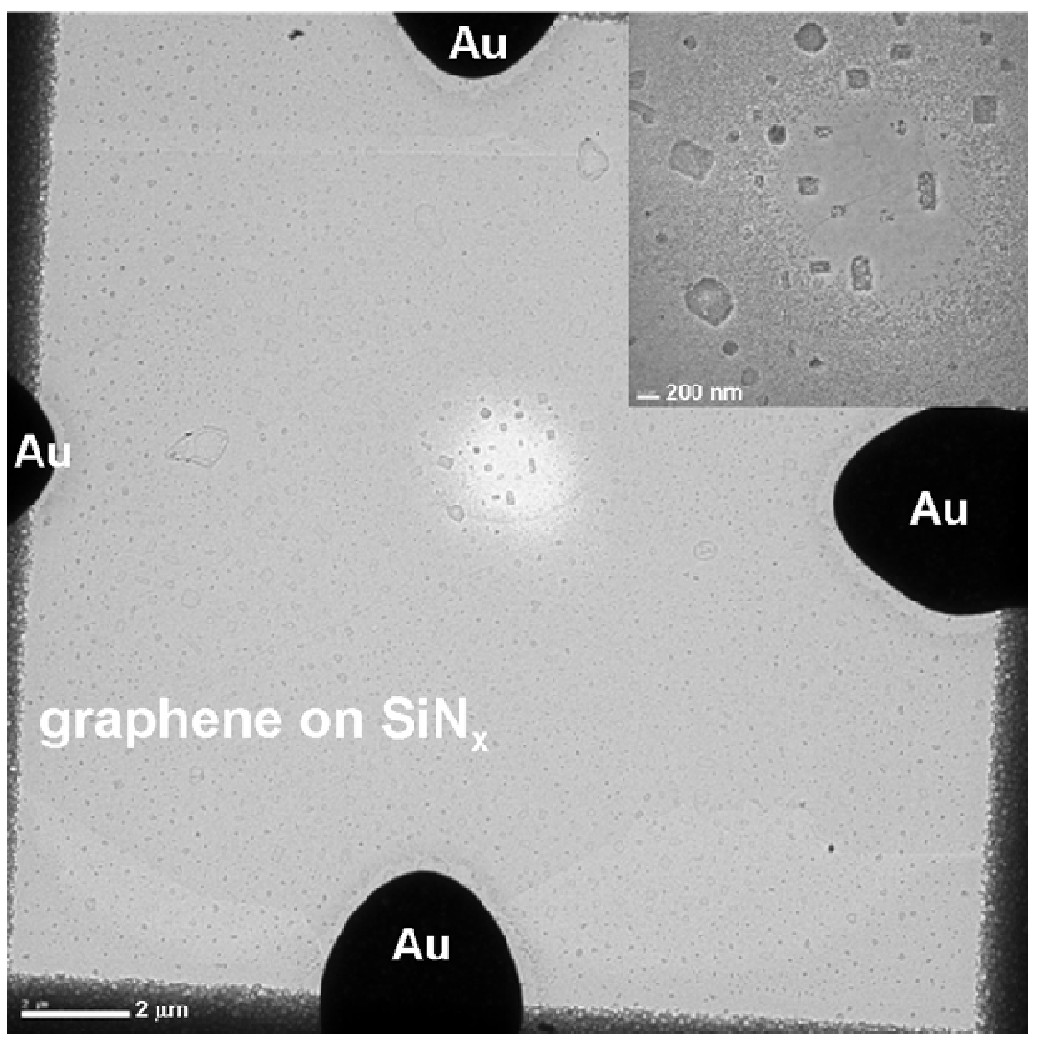

no bias
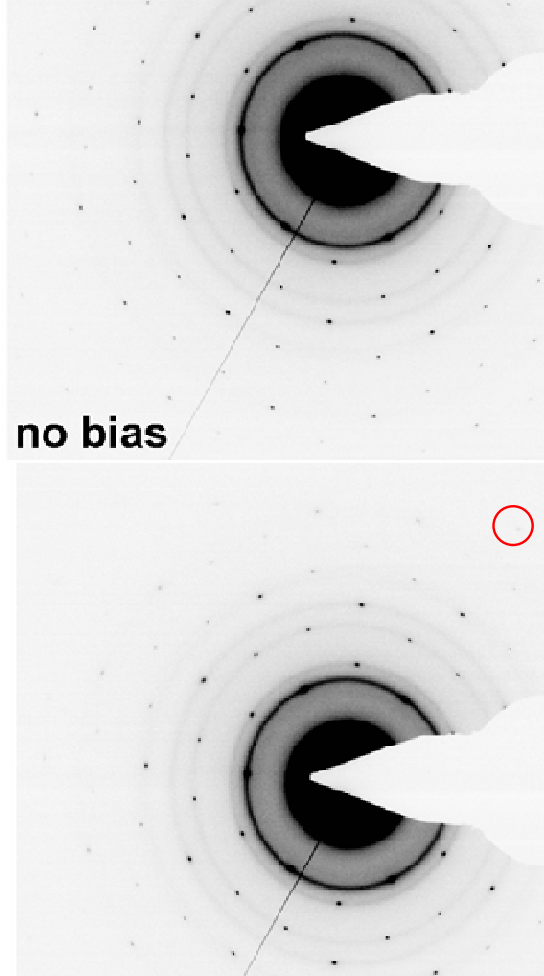

$3 \mathrm{~V}$ bias

Figure 1. (left) Bright-field TEM image of graphene transferred to a biasing chip. The "hot spot" in the center (higher magnification in the inset) formed when residual $\mathrm{SiO} 2$ and contaminants sublimated from the membrane upon heating. (right) SAED pattern acquired from the hot spot. The same peak is encircled in red in each pattern to highlight the attenuation of higher-order peaks upon heating. 